\title{
Theoretical chemistry of superheavy elements: Support for experiment
}

\author{
Valeria Pershina ${ }^{\mathrm{a}}$ \\ GSI Helmholtzzentrum für Schwerionenforschung GmbH, 64291 Darmstadt, Germany
}

\begin{abstract}
Spectacular developments in the relativistic quantum theory and computational algorithms in the last few decades allowed for accurate calculations of properties of the superheavy elements (SHE) and their compounds. Often conducted in a close link to the experimental research, these investigations helped predict and interpret an outcome of sophisticated and expensive experiments with single atoms and to reveal the magnitude and importance of relativistic effects.
\end{abstract}

\section{Importance of chemical theory for SHE}

Conceptually, it is the atomic number $Z$ and ground state electronic configuration of an element that define its position in the Periodic Table. However, information on chemical behaviour of a new element with respect to its homologs often serves as an additional proof of the proper assignment $[1,2]$. This is particularly important for superheavy elements (SHE): It is meanwhile well established that relativistic effects on their electron shells are so strong that deviations from trends in chemical properties observed within lighter homologs in the groups can occur [3]. Important properties to assess similarity of SHE with the lighter congeners are atomic/ionic radii, redox potentials and complex formation.

The atomic number of many SHE can be determined by physical methods, while the electron configuration can currently be provided only by theory. Experimental studies of chemical properties of short-lived SHE isotopes are also limited to measurements of very few properties, mainly, volatility of atoms or molecules using gas-phase chromatography [4], or complex formation using liquid-phase separation techniques [5]. (See also the contribution of A. Türler in this volume). Knowledge of many other important properties, such as chemical composition, stability, geometrical configuration, ionization potentials (IP), or electron transitions, can only be provided by theory. Thus, in the area of SHE, theoretical studies become extremely important and are often the only source of useful chemical information [6-9]. They are also invaluable for predicting and/or interpreting the outcome of sophisticated and demanding experiments with single atoms. Moreover, since nature is relativistic, it is only theory that can demonstrate the importance and magnitude of relativistic effects by comparing the observed behaviour with that predicted on the basis of relativistic vs. non-relativistic calculations.

\footnotetext{
a e-mail: v.pershina@gsi.de
} 

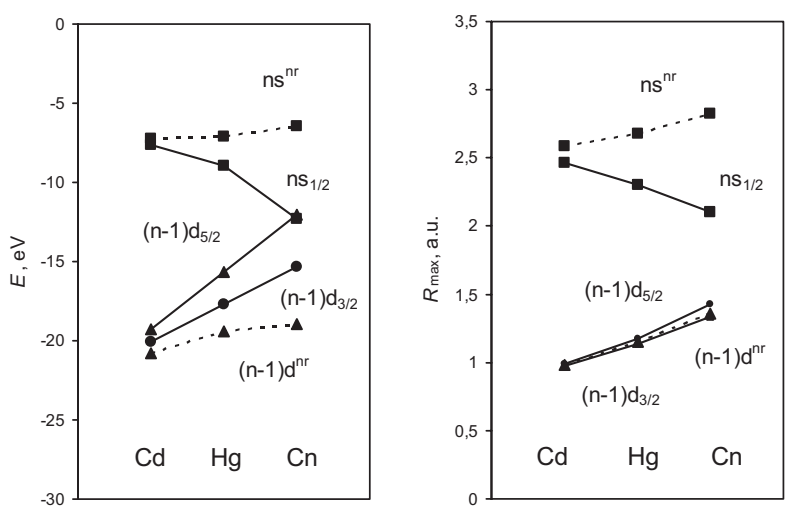

Figure 1. Relativistic (solid line) and nonrelativistic (dashed line) energy, $E$, and the maximum of the radial charge density, $R_{\max }$, of the group-12 valence $\mathrm{n} s$ and (n-1)d AOs [6].

\section{Relativistic and QED effects for SHE}

With increasing $\mathrm{Z}$, the velocity of an electron increases. The Bohr model for a hydrogenlike species gives it as $v=\left(2 \pi e^{2} / n h\right) Z$. As a result, the relativistic mass of an electron also increases

$$
m=m_{0}\left[\left(1-(v / c)^{2}\right]^{-1 / 2},\right.
$$

where $m_{0}$ is the mass at zero velocity (rest mass) and $c$ is the speed of light. From the sixth row onwards, the $m / m_{0}$ ratio exceeds by $10 \%$, so that relativistic effects cannot be neglected anymore. For example, for Fl, $m / m_{0}=1.79$, and it is 1.95 for element 118 . As a result of the relativistic mass increase, the electron (negative) energy increases and the orbital radius shrinks according to

$$
\begin{gathered}
E=-\left(2 \pi^{2} e^{4} / n^{2} h^{2}\right) m Z^{2}, \\
r=Z e^{2} / m v^{2},
\end{gathered}
$$

where $n$ is the principal quantum number, $e$ is the charge of the electron, and $h$ is Planck's constant. The contraction (Eq. (3)) and stabilization (Eq. (2)) of the hydrogen-like $s$ and $p_{1 / 2}$ electrons is a direct relativistic effect and it was shown to originate from the inner $K$ - and $L$ shell regions. The second (indirect) relativistic effect causes an expansion and destabilization of the $p_{3 / 2}, d, f$, and $g$ atomic orbitals (AOs) due to increased screening of the nucleus by the relativistically contracted $s$ and $p_{1 / 2}$ AOs. The third relativistic contribution comes from the spin-orbit (SO) splitting of the AOs with $l>0$. All the three effects change approximately as $Z^{2}$ for the valence shells down a column of the Periodic Table.

Figure 1 shows, e.g., relativistic effects on group-12 elements: the $n s$ AO stabilization reaches $5.8 \mathrm{eV}$ for $\mathrm{Cn}$, which is the maximum in the $7^{\text {th }}$ row of the Periodic Table, and its contraction is $25 \%$; the $\mathrm{SO}$ splitting of the $5 d \mathrm{AOs}$ is also large, e.g., $3.3 \mathrm{eV}$ for $\mathrm{Cn}$. The $\mathrm{SO}$ splitting of the $7 p$ AOs reaches $11.8 \mathrm{eV}$ at element 118. For the heavier elements, relativistic effects on their valence orbitals are even more pronounced and could lead to properties very different from those of the lighter homologs.

Breit effects (accounting for magnetic and retardation interactions) on valence orbital energies and IPs of the heaviest elements are small, for example, only $0.02 \mathrm{eV}$ for element 121. Quantum electrodynamic (QED) effects such as vacuum polarization and electron 
self-energy can reach a few \% in IP, electron affinities (EA), or transition energies for SHE (see, for instance, Refs. [10, 11]).

\section{Methods to calculate electronic structures of SHE}

There are several issues devoted to the relativistic methods (see, for example, [12]). It is meanwhile established that for highly relativistic SHE systems, the most appropriate approaches are those that treat both relativity and electron correlation at the highest possible level of theory: both effects were shown to contribute to more than $50 \%$ to binding energies and other properties of the $7^{\text {th }}$-row elements. Presently the highest theoretical level for the many-body methods is the Dirac-Coulomb-Breit (DCB) Hamiltonian

$$
h_{D C B}=\sum_{i} h_{D}(i)+\sum_{i<j}\left(1 / r_{i j}+B_{i j}\right)
$$

where $B_{i j}$ is the Breit term and the Dirac-Coulomb one-electron Hamiltonian is

$$
h_{D}(i)=c \vec{\alpha}_{i} \vec{p}_{i}+c^{2}\left(\beta_{i}-1\right)+V^{n}(i) .
$$

It contains the one-electron Dirac Hamiltonian plus the nuclear potential, $V^{n}$, and the operator $1 / r_{i j}$ for the Coulomb interaction between electrons. The wave function is a four-component (4c) vector (spinor). The $V^{n}$ includes the effect of the finite nuclear size, while some finer effects, like QED, can be added to $h_{D C B}$ self-consistently for atoms, or perturbatively for molecules. Correlation effects are taken into account by configuration interaction (CI), manybody perturbation theory (MBPT) or, presently at the highest level of theory, coupled cluster theory with single-double (and perturbative triple) excitations (CCSD(T)) or Fock-Space (FS) $\mathrm{CC}$ theory.

Wavefunction based (ab-initio) methods solving the Dirac many-electron Eq. (4) are the most accurate. Nevertheless, the problems of electron correlation and proper basis sets make the usage of the 4c-DF methods very limited in molecular calculations. These methods are still too computer time intensive. Therefore, almost equally accurate $2 \mathrm{c}$-methods have recently been developed.

Effective core potentials (ECP) allow for more economic calculations within the DHF schemes by replacing the inner core orbitals, which do not take part in the bond formation, by a special (effective core) potential. In this way, the number of basis functions and, therefore, two-electron integrals, is drastically diminished. There are two main types of the ECPs, pseudo potentials (PP) and model potentials (MP).

Relativistic density functional theory (DFT). Due to the high accuracy and efficiency, computational schemes based on the 4c- and 2c-DFT methods are among the most popular in theoretical chemistry, especially for extended systems, large molecules, liquids, or solids. The accuracy depends on the adequate choice of the exchange-correlation potential, $E^{\text {ex }}$, whose exact form is unknown. There is quite a number of the GGA $E^{\mathrm{ex}}$ and their choice is dependent on the system. For solid-state and adsorption calculations of SHE, the ADF BAND program is very promising [13].

\section{Atomic properties of SHE}

\subsection{Electronic configurations}

Earlier predictions of chemical properties of elements $Z=104$ through $Z=172$ were made on the basis of Dirac-Fock (DF) and Dirac-Slater (DS) atomic calculations (see [3] for a review). Later, atomic properties of SHE were calculated using the MCDF and DCB 
Table 1. Ground states predicted using various relativistic atomic methods ${ }^{\mathrm{a}}$.

\begin{tabular}{llllll}
\hline Element & $\begin{array}{l}\text { Madelung } \\
\text { principle }\end{array}$ & DS & DF & MCDF & DCB CC \\
\hline & $\begin{array}{l}\text { Seaborg } \\
(1968)\end{array}$ & $\begin{array}{l}\text { Waber et al. } \\
(1968)\end{array}$ & $\begin{array}{l}\text { Fricke et al. } \\
(1971)\end{array}$ & $\begin{array}{l}\text { Nefedov et al. } \\
(2006)\end{array}$ & $\begin{array}{l}\text { Eliav et al. } \\
(1998)\end{array}$ \\
\hline 121 & $7 d$ & $7 d$ & $8 p_{1 / 2}$ & $8 p_{1 / 2}$ & $8 p_{1 / 2}$ \\
122 & $5 g^{2}$ & $7 d^{2}$ & $7 d 8 p_{1 / 2}$ & $7 d 8 p_{1 / 2}$ & $7 d 8 p_{1 / 2}$ \\
123 & $5 g^{3}$ & $6 f^{1} 7 d^{2}$ & $6 f 7 d 8 p_{1 / 2}$ & $6 f^{2} 8 p_{1 / 2}$ & $\ldots$ \\
\hline
\end{tabular}

${ }^{a}$ See $[3,6,7,9]$ for exact references.

FSCC/CCSD(T) methods, with the heaviest element treated in this way being $Z=122$ [9]. Table 1 shows the progress in the predictive power of the atomic methods upon their development for ground-state configurations of elements 121, 122, and 123.

One can see that the calculated states deviate from those predicted via the simple Madelung's principle. In the area of $Z>122$, the elements are characterized by mixing of configurations coming from the partially filled $8 p_{1 / 2,3 / 2}, 7 d_{3 / 2,5 / 2}, 6 f_{5 / 2,7 / 2}$ and $5 g_{7 / 2,9 / 2}$, etc. shells. The proximity of the valence SO bands makes the search for the correct ground state very difficult. The usual classification on the basis of a simple electronic configuration and the placement of these elements in this part of the Periodic Table becomes, therefore, problematic, so that the shape of the table at these high $Z$ is still under discussion and debate $[3,14,15]$.

\subsection{Spectroscopic properties and predictions of SHE transport}

Ionization potentials in the DF and DS approximations are available for SHE up to $Z=166$ [3], and in the MCDF and DCB CC approximation till $Z=122$ [9]. Multiple IPs were also calculated at the MCDF level of theory for elements Rf through Hs. They were used for estimates of stabilities of various oxidation states of these elements (see [6]).

Electron affinities were also calculated for quite a few of the heaviest elements at the DCB $\mathrm{CC}$ level of theory. Fl, has, e.g., no EA. On the contrary, element 118 has a positive EA of $0.058 \mathrm{eV}$, shown by the DCB FSCC + QED calculations. Both unusual results were shown to be due to the influence of relativistic effects.

Atomic/ionic/covalent radii and polarizabilities of SHE are now also available at various levels of theory $[2,6,7,14]$. An important finding is smaller covalent radii of the $6 d$ elements than those of the $5 d$ ones in group 9 through 12 , called a "transactinide break" $[14,15]$.

The atomic data were useful in predicting chemical behaviour of SHE [3, 6, 7], such as transport through Teflon capillaries from an accelerator to the chemistry set up, and/or their interaction with inert materials of gas-phase chromatography column. For that purpose, the following adatom-slab model for the adsorption energy, $E_{\text {ads }}$, was used:

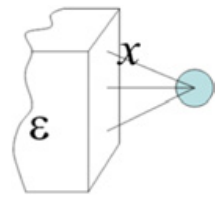

$$
E(x)=-\frac{3}{16}\left(\frac{\varepsilon-1}{\varepsilon+2}\right) \frac{\alpha_{m o l}}{\left(\frac{1}{I P_{\text {slab }}}+\frac{1}{I P_{m o l}}\right) x^{3}} .
$$

Here, $\varepsilon$ is the dielectric constant of the adsorbent material and $x$ is the adatom-surface distance related to the van der Waals radius of the species. Table 2 shows two examples of such predictions for very different cases: for Fl with a small $\alpha$ and a high IP, and for element 120 with a large $\alpha$ and a small IP. The obtained low value of $E_{\text {ads }}$ of Fl is indicative of its easy transport through Teflon capillaries to the chemistry set up, which was not obvious for 
Table 2. DC CCSD(T) calculated atomic properties of Fl $\left(7 s^{2} 7 p_{1 / 2}{ }^{2}\right)$ and of element $120\left(8 s^{2}\right)$, and their adsorption enthalpies, $-\Delta H_{\text {ads }}$, on Teflon [7].

\begin{tabular}{lllll}
\hline Element & $\alpha$, a.u. & $\mathrm{IP}, \mathrm{eV}$ & $R_{\mathrm{vdW}}, \AA$ & $-\Delta H_{\mathrm{ads}}, \mathrm{kJ} / \mathrm{mol}$ \\
\hline Fl & 29.5 & 8.539 & 3.94 & 10.4 \\
120 & 29.5 & 8.539 & 3.94 & 10.4 \\
\hline
\end{tabular}

element 120 . Using the calculated properties and equation 6 , a yield $N / N_{0}\left(N_{0}\right.$ is the number of atoms entering the column or capillary, $N$ is the number at the exit) of a hypothetical ${ }^{300} 120$ of $90 \%$ at the end of a Teflon capillary of $1 \mathrm{~m}$ was then determined. Thus, this element should also be transported, and the limiting factor is its half-life [7].

\section{Predictions of experimental behaviour of SHE}

\subsection{Gas-phase experiments}

Group 4-8 molecules. Gas-phase chromatography experiments provide measurements of volatility of SHE as adsorption temperature, $T_{\mathrm{ads}}$, on a surface of detectors of the chromatography column usually covered with $\mathrm{SiO}_{2}$ or gold layers [2, 4]. Obtained on their basis the adsorption enthalpy, $\Delta H_{\text {ads }}$, is then used to deduce the sublimation enthalpy, $\Delta H_{\text {sub }}$, being a measure of volatility in macrochemistry, using an empirical correlation between these quantities. The task of the theory was to predict both $\Delta H_{\text {ads }}$ and $\Delta H_{\text {sub }}$ and to see whether such a correlation is valid in the area of the heaviest elements.

For weak interactions of closed-shell SHE atoms or molecules with inert surfaces, no modern DFT methods can provide reliable values of $E_{\text {ads }}$ : there are no dispersioncorrected $E^{\text {ex }}$ for SHE, as they should be based on empirical data. Thus, for those systems, adatom/molecule-slab models proved to be useful. For molecules without dipole moments, Eq. 6 was used, while for those with dipole moments, $\mu$, the following equation:

$$
E(x)=-\frac{2 Q e \mu_{m o l}{ }^{2}}{x^{2}}-\frac{Q^{2} e^{2} \alpha_{m o l}}{2 x^{4}}-\frac{3}{2} \frac{\alpha_{m o l} \alpha_{C l}}{\left(\frac{1}{I P_{m o l}}+\frac{1}{I P_{C l}}\right) x^{6}} .
$$

All the properties of group-4 through 8 molecules used in Eqs. (6) and (7) have accurately been calculated via 4c-DFT and PP CC methods (see Tables 8 and 9 in [6]). Thus, e.g., for the prediction of adsorption of group-7 oxychlorides $\mathrm{MO}_{3} \mathrm{Cl}(\mathrm{M}=\mathrm{Tc}, \mathrm{Re}$, and $\mathrm{Bh})$ on a chlorinated quartz surface, equation 7 was used [6]. For an effective charge $Q=$ -0.4 of the surface chlorine ions, $-\Delta H_{\mathrm{ads}}=(48 \pm 2) \mathrm{kJ} / \mathrm{mol}$ and $-\Delta H_{\mathrm{ads}}=(78 \pm 5)$ $\mathrm{kJ} / \mathrm{mol}$ for $\mathrm{TcO}_{3} \mathrm{Cl}$ and $\mathrm{BhO}_{3} \mathrm{Cl}$, respectively, were obtained with respect to the measured $-\Delta H_{\text {ads }}\left(\mathrm{ReO}_{3} \mathrm{Cl}\right)=(61 \pm 2) \mathrm{kJ} / \mathrm{mol}$. These $-\Delta H_{\text {ads }}$ of the Tc and $\mathrm{Bh}$ oxychlorides were confirmed by experiment giving $(51 \pm 2) \mathrm{kJ} / \mathrm{mol}$ and $\left(75_{-9}^{+6}\right) \mathrm{kJ} / \mathrm{mol}$, respectively (see [2]). The sequence in volatility, $\mathrm{TcO}_{3} \mathrm{Cl}>\mathrm{ReO}_{3} \mathrm{Cl}>\mathrm{BhO}_{3} \mathrm{Cl}$, was revealed and explained by increasing $\mu$ in this row.

Another example deals with predictions of volatility of group- 8 tetroxides, $\mathrm{MO}_{4}(\mathrm{M}=\mathrm{Ru}$, Os, and $\mathrm{Hs}$ ). Their adsorption on $\mathrm{SiO}_{2}$, or a silicon nitride surface was predicted with the use of Eq. (6) and 4c-DFT calculated molecular properties (Table 3).

Thermochromatography gas-phase experiments have, indeed, shown that $-\Delta H_{\mathrm{ads}}\left(\mathrm{HsO}_{4}\right)$ is only $6 \mathrm{~kJ} / \mathrm{mol}$ larger than $-\Delta H_{\text {ads }}\left(\mathrm{OsO}_{4}\right)$ (see [2] for the reference), in excellent agreement with the calculations (Table 3). Such a reversal of the trend in volatility in the group could not be predicted from a simple extrapolation, but came out as a result of the accurate calculations and precise measurements. 
Table 3. Ionization potentials, IP (in eV), polarizabilities, $\alpha$ (in a.u.), bond lengths, $R_{e}$ (in $\AA$ ), and adsorption enthalpies, $-\Delta H_{a d s}$, (in $\mathrm{kJ} / \mathrm{mol}$ ), of $\mathrm{MO}_{4}(\mathrm{M}=\mathrm{Ru}$, Os, and $\mathrm{Hs}$ ) on quartz .

\begin{tabular}{llllc}
\hline & Meth. & $\mathrm{RuO}_{4}$ & $\mathrm{OsO}_{4}$ & $\mathrm{HsO}_{4}$ \\
\hline $\mathrm{IP}$ & calc. & 12.21 & 12.35 & 12.29 \\
& exp. & 12.19 & 12.35 & - \\
$\alpha$ & calc. & 58.07 & 55.28 & 65.99 \\
& exp. & 58.64 & 55.13 & - \\
$R_{\mathrm{e}}$ & calc. & 1.712 & 1.719 & 1.779 \\
& exp. & 1.706 & 1.711 & - \\
$-\Delta H_{\text {ads }}$ & calc. & $41.0 \pm 1$ & $39.0 \pm 1$ & $45.4 \pm 1$ \\
& exp. & - & $39 \pm 1$ & $46 \pm 2$ \\
\hline
\end{tabular}

${ }^{\mathrm{a}}$ See $[2,6,7]$ for references.
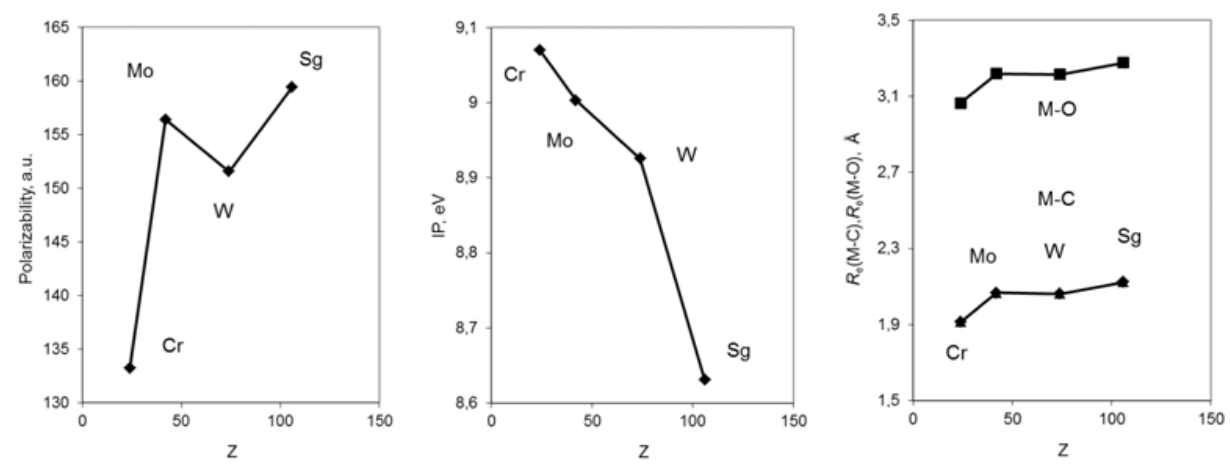

Figure 2. Calculated ionization potentials (IP), polarizabilities $(\alpha)$ and equilibrium bond lengths $\left(R_{\mathrm{e}}\right)$ of $\mathrm{M}(\mathrm{CO})_{6}(\mathrm{M}=\mathrm{Cr}, \mathrm{Mo}, \mathrm{W}$, and $\mathrm{Sg})[17]$.

Another example deals with recent successful predictions of volatility of group-6 carbonyls. First of all, stability of $\operatorname{Sg}(\mathrm{CO})_{6}$ had to be known. A work [16] based on the CCSD RECPs calculations have shown that the first bond dissociation energy (FBDE), $\mathrm{M}(\mathrm{CO})_{6} \rightarrow \mathrm{M}(\mathrm{CO})_{5}+\mathrm{CO}$, should increase in group 6, reaching $2.20 \mathrm{eV}$ for $\mathrm{Sg}(\mathrm{CO})_{6}$. The $\mathrm{Sg}(\mathrm{CO})_{6}$ was, indeed, synthesised and experiments are on the way to measure its FBDE.

To predict volatility of $\mathrm{Sg}(\mathrm{CO})_{6}$, the $4 \mathrm{c}$-DFT calculations were performed for the group-6 carbonyls and $E_{\text {ads }}$ were estimated using the adatom-slab model (Eq. (6)) [17]. The trends in the calculated molecular properties are shown in Fig. 2 reflecting, e.g., peculiarities in $R_{\mathrm{e}}$ and $\alpha$ caused by the lanthanide contraction from Mo to W. The $-\Delta H_{\text {ads }}$ of $(46.2 \pm 2.5) \mathrm{kJ} / \mathrm{mol}$ of $\mathrm{Sg}(\mathrm{CO})_{6}$ was predicted to be almost equal to the measured $-\Delta H_{\text {ads }}$ of $\mathrm{W}(\mathrm{CO})_{6}$ of $(46.5 \pm$ $2.5) \mathrm{kJ} / \mathrm{mol}$. The experimental $\Delta H_{\text {ads }}$ have, indeed, indicated an almost equal volatility of the $\mathrm{W}$ and $\mathrm{Sg}$ carbonyls giving $-\Delta H_{\mathrm{ads}}$ of $\mathrm{Sg}(\mathrm{CO})_{6}$ of $(50 \pm 4) \mathrm{kJ} / \mathrm{mol}$ [18], in very good agreement with the calculations [17].

Elements 112 and 114. Another exciting case was a study of volatility of $\mathrm{Cn}$ and $\mathrm{Fl}$, having closed-shell $\left(6 d^{10} 7 s^{2}\right)$ and quasi-closed shell $\left(7 s^{2} 7 p_{1 / 2}{ }^{2}\right)$ ground states, respectively. Due to the strong relativistic effects on their AOs (Fig. 1), these elements were expected to be extremely inert and volatile. Also their extrapolated $\Delta H_{\text {sub }}$ are the smallest in the corresponding groups.

Experimentally, volatility of $\mathrm{Cn}$ in comparison with that of $\mathrm{Hg}$ and $\mathrm{Rn}$ was to be studied as adsorption process on a gold surface of detectors of a gas-phase thermochromatography column [19-21]. The questions to the electronic structure theory, therefore, were: are $\mathrm{Cn}$ and Fl metallic in the solid state, or are they more like a solid noble gas? How volatile and reactive towards gold are $\mathrm{Cn}$ and $\mathrm{Fl}$ atoms in comparison with the lighter homologs and with $\mathrm{Rn}$ ? 

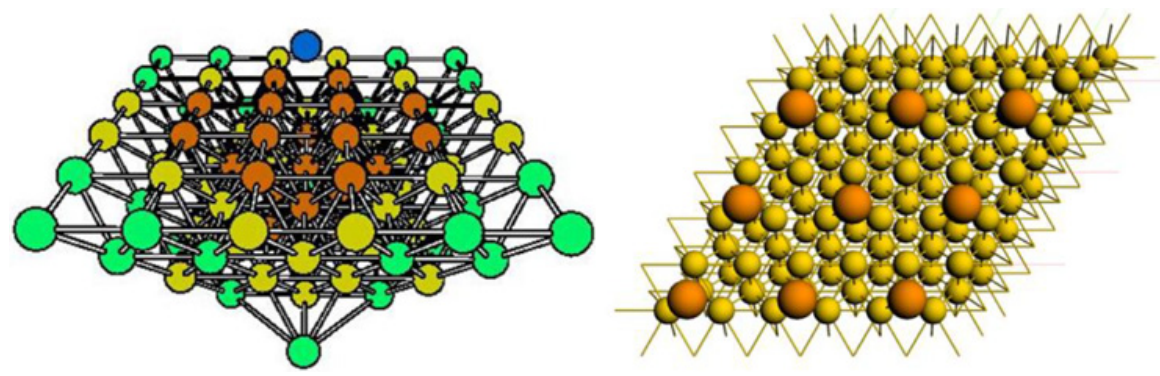

Figure 3. Models simulating adsorption of $\mathrm{M}$ on a $\mathrm{Au}(111)$ surface. The left panel is a cluster model treated via a molecular code. The right panel is a super-cell one treated via a periodic code.

Table 4. Adsorption enthalpies, $-\Delta H_{\text {ads }}$ (in $\mathrm{kJ} / \mathrm{mol}$ ), of $\mathrm{Hg}, \mathrm{Cn}, \mathrm{Pb}$ and $\mathrm{Fl}$ on a hydroxylated quartz and a gold (111) surface at very low coverage calculated using the ADF BAND periodic and 4c-DFT cluster approaches for single species in comparison with experiment.

\begin{tabular}{lllllll}
\hline Surface & $\mathrm{Hg}$ & $\mathrm{Cn}$ & $\mathrm{Pb}$ & $\mathrm{Fl}$ & Method & References \\
\hline $\mathrm{SiO}_{2}$ & 54 & 26 & 152 & 21 & ADF BAND & {$[25]$} \\
& $42 \pm 2$ & - & $165 \pm 4$ & - & Experiment & see [2] \\
$\mathrm{Au}$ & $78^{\mathrm{a}}$ & 45 & 240 & 68 & BAND/4c-DFT & {$[23,24]$} \\
& $98 \pm 3$ & $52_{-3}^{+4}$ & 234 & $34_{-11}^{+54} ; \geq 48$ & Experiment & {$[19,21]$} \\
\hline
\end{tabular}

a Preliminary ADF BAND value for the non-ideal surface.

The 4c-DFT calculations of the binding energies of group-12 homonuclear dimers $[6,7]$ and the scalar relativistic (SR) DFT periodic ones of cohesive energies [22] have shown $\mathrm{Cn}-\mathrm{Cn}$ and the bulk of $\mathrm{Cn}$ to be more bound than the corresponding $\mathrm{Hg}$ systems. Thus, a decrease in $\Delta H_{\text {sub }}$ in group 12 should not continue with $\mathrm{Cn}$. In contrast to the M-M-bonding, the $\mathrm{M}$-Au one should be weaker for $\mathrm{Cn}$ than for $\mathrm{Hg}$ due to the stronger relativistic stabilization of the $7 s(\mathrm{Cn}) \mathrm{AO}$ [23]. This means that $-\Delta H_{\mathrm{ads}}(\mathrm{Hg})>-\Delta \mathrm{H}_{\mathrm{ads}}(\mathrm{Cn})$, which is opposite to $\Delta H_{\text {sub }}(\mathrm{Hg})<\Delta H_{\text {sub }}(\mathrm{Cn})$. Thus, there is no correlation between $-\Delta H_{\text {ads }}$ and $\Delta H_{\text {sub }}$ in group 12. The Fl-Au bond was also shown to be weaker than the $\mathrm{Pb}-\mathrm{Au}$ one, but stronger than the $\mathrm{Cn}-\mathrm{Au}$ one. Numerous calculations for the $\mathrm{Cn} / \mathrm{Fl}-\mathrm{Au}_{n}$ systems, where $\mathrm{Au}_{n}$ are clusters simulating a gold surface (Fig. 3, left), have come to the same result, $E_{b}(\mathrm{Fl}-\mathrm{Au})>E_{b}$ (Cn-Au) (Table 4).

Experiments [19], being in good agreement with the theoretical predictions for adsorption of $\mathrm{Cn}$ on gold [23], turned out to be in some disagreement with each other and partially with the theory for adsorption of Fl [20,21] (Table 4). Therefore, further experiments are required to shed more light on this interesting case.

For element 113, to be experimentally studied next, the calculations have shown that it should be much more reactive than $\mathrm{Cn}$ and $\mathrm{Fl}$ due to one unpaired $7 p_{1 / 2}$ electron, so that its $-\Delta H_{\text {ads }}$ on gold of $150 \mathrm{~kJ} / \mathrm{mol}$ should be larger than $-\Delta H_{\text {ads }}$ of $\mathrm{Cn}$ and Fl (Table 4) [7]. In group 13 the trend to an increase in volatility should continue with element 113: its weaker adsorption on gold is a result of the $7 p_{1 / 2}$ AO SO stabilization and, therefore, weaker bonding.

Calculations of $E_{\text {ads }}(\mathrm{M}$-gold) using the ADF BAND code are in progress [24] (Fig. 3, right). Modelling non-ideal surfaces (with vacancies, steps) allowed for reaching even better agreement of the calculated $E_{\text {ads }}$ of $\mathrm{Hg}$ and $\mathrm{Pb}$ with their experimental $-\Delta H_{\text {ads }}^{A u}$ (see Table 4). Results for SHE will follow.

Assistance was also rendered to experiments on volatility of $\mathrm{Hg} / \mathrm{Cn}$ and $\mathrm{Pb} / \mathrm{Fl}$ with the use of $\mathrm{SiO}_{2}$ covered detectors located ahead of the gold-plated ones in a chromatography column (allowing for differentiating between $\Delta H_{\text {ads }}$ on surfaces of these different materials). 


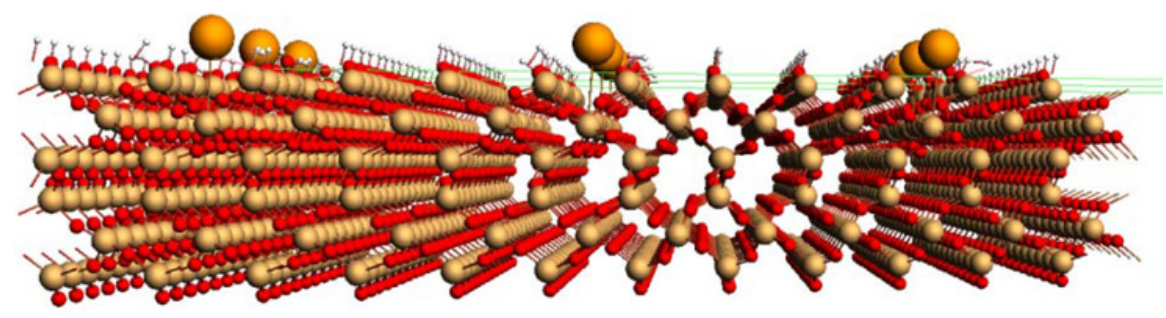

Figure 4. A supercell modelling adsorption of $\mathrm{M}$ on vicinal silanols for low adsorbate coverage. Reproduced from [25] with permission from the PCCP Owner Societies.

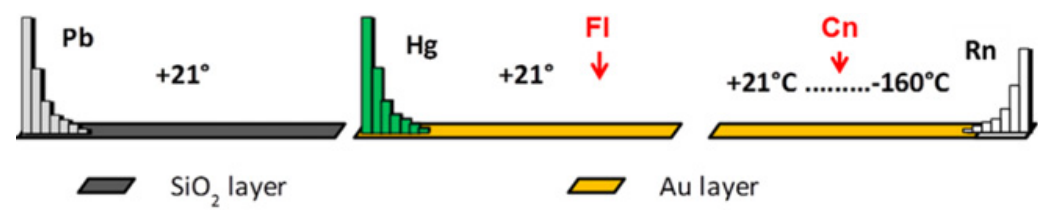

Figure 5. Predicted adsorption of $\mathrm{Cn}$ and $\mathrm{Fl}$ on quartz and gold surfaces of the chromatography column. Reproduced from [25] with permission from the PCCP Owner Societies.

Accordingly, periodic SR and SO calculations were performed on $E_{\text {ads }}$ of these elements on a quartz surface using the ADF BAND code [25]. Two modifications of the hydroxylated $\mathrm{SiO}_{2}$ surfaces were considered: vicinal $(80 \%)$ and germinal $(20 \%)$ silanols. A $(4 \times 4)$ super-cell modelling adsorption on a vicinal silanol for very low coverage is shown in Fig. 4.

Thus, according to the calculations [25], $\mathrm{Pb}$ should adsorb on the $\mathrm{SiO}_{2}$ surface at room temperature, while $\mathrm{Hg}, \mathrm{Cn}$, and $\mathrm{Fl}$ should not. The latter three elements should adsorb, however, on gold, with $\mathrm{Hg}$ revealing much stronger interaction (Table 4 and Fig. 5). The reason for the non-interaction of $\mathrm{Cn}$ and $\mathrm{Fl}$ with quartz and weaker interaction with gold than that of $\mathrm{Hg}$ and $\mathrm{Pb}$, respectively, is the strongest relativistic effects on their valence electrons making these elements rather inert. Experiments have, indeed, revealed that $\mathrm{Cn}$ and $\mathrm{Fl}$ do not adsorb on $\mathrm{SiO}_{2}\left(\Delta H_{\text {ads }}\right.$ to be published).

The theory has also considered interaction of even heavier elements such as 115 through 120 with gold and other metals of interest on the basis of Au-containing dimers [6,7]. These results might be of interest for future vacuum chromatography experiments, provided sufficiently long-living isotopes of these elements are found.

Some molecular species of even heavier elements are suggested in [14], whose stability may be proven by detailed calculations.

\subsection{Aqueous chemistry experiments}

Complex formation is known to increase in the groups at the beginning of the $3 d-5 d$ - series. It was, therefore, important to establish whether this trend is continued with the $6 d$-elements. Experimental investigations were accordingly devoted to the study of the complex formation of Rf, $\mathrm{Db}$, and $\mathrm{Sg}$ (elements with sufficiently long half-lives) in aqueous solutions of $\mathrm{HF}, \mathrm{HCl}$, $\mathrm{HBr}$, and $\mathrm{H}_{2} \mathrm{SO}_{4}$ acids. Liquid-liquid extraction, or sorption chromatography separations were applied to the studied species $[2,5]$. To render assistance to those experiments, predictions of complex-formation constants and distribution coefficients, $K_{\mathrm{d}}$, between an aqueous and organic phases (or resins) were made with the use of a model that treats a 
free energy change of a complex formation reaction as a sum of the ionic and covalent constituents. The latter are obtained via 4c-DFT electronic structure calculations of the systems of interest $[6,7]$.

The results have demonstrated that even though the heaviest elements are homologs of their lighter congeners in the chemical groups, trends in the complex formation are not necessarily continued with them. (See, e.g., predictions of an unexpected trend in the $K_{\mathrm{d}}$ values of the group- 5 complexes by extraction from the $\mathrm{HCl}$ solutions into amines, confirmed by experiments [5].) The calculations have also shown that simple models based on the ratio of the cation charge to its size, or extrapolations are unreliable in the area of SHE. Only by performing relativistic calculations of the real chemical equilibria, can the complex formation, or hydrolysis be correctly predicted.

A summary of the predicted trends in hydrolysis, complex formation and extraction of the group- 4 through 6 elements including the heaviest is given in $[6,7]$. As one can see there, most of the predictions have been confirmed by the experiments, while some of them, like predictions for $\mathrm{Sg}$ in HF solutions, are still awaiting confirmation.

\section{Summary}

A large number of theoretical chemical studies were performed on the heaviest elements and their compounds such as molecules, aqueous complexes, and solid-state systems. They were based on the accurate calculations with the use of the most advanced relativistic methods, such as $4 \mathrm{c} / 2 \mathrm{c}$-DFT, PP and DF CC ones. With many of them carried out in a close link to the experimental research, these studies have contributed to better understanding of the chemistry of these exotic elements, and the role and magnitude of relativistic effects. A synergy between theoretical and experimental research has been achieved.

The results have demonstrated that even though the heaviest elements are homologs of their lighter congeners in the chemical groups, trends in properties are not necessarily continued with them. Thus, in this area of the Periodic Table simple extrapolations or semiempirical models turned out to be unreliable. Relativistic calculations at the highest level of theory are, therefore, mandatory to achieve convincing results.

Relativistic theory will undergo further methodical developments like, e.g., creation of special techniques in atomic CC methods to treat open shell systems (needed for predictions of electronic configurations of elements with $Z>122$ ), or inclusion of QED effects on the SCF basis in molecular calculations. Also, dispersive potentials for SHE should be worked out in DFT methods in order to treat more accurately weak interactions.

For elements heavier than $Z=118$, theoretical investigations will be very exciting, since resemblance of properties with their lighter homologs in the groups is expected to be even less pronounced.

The author thanks her former and present collaborators J. Anton, T. Bastug, E. Eliav, U. Kaldor, A. Borschevsky, and M. Ilias for the fruitful joint work. She also appreciates valuable discussions of experimental results with A. Yakushev, J.V. Kratz, Ch. E. Düllmann, A. Türler, and R. Eichler.

\section{References}

[1] The Chemistry of the Superheavy Elements, 2nd edition, eds. M. Schädel and D. Shaughnessy (Springer, Heidelberg, 2014).

[2] A. Türler and V. Pershina, Chem. Rev. 113, 1237 (2013).

[3] B. Fricke, Struct. Bond. 21, 89 (1975). 
[4] H.W. Gäggeler and A. Türler, Gas-Phase Chemistry of Superheavy Elements. In reference [1], p. 415.

[5] J.V. Kratz and Y. Nagame, Liquid-Phase Chemistry of Superheavy Elements. In reference [1], p. 309.

[6] V. Pershina, Theoretical Chemistry of the Heaviest Elements. In reference [1], p. 135.

[7] V. Pershina, Nucl. Phys. A 944, 578 (2015).

[8] P. Schwerdtfeger and M. Seth, In: Encyclopedia on Calculational Chemistry (Wiley, New York, 1998) Vol. 4, p. 2480.

[9] E. Eliav, S. Fritzsche, U. Kaldor, Nucl. Phys. A 944, 518 (2015).

[10] P. Pyykkö, Chem. Rev. 88, 563 (1988).

[11] P. Schwerdtfeger et al., Nucl. Phys. A 944, 551 (2015).

[12] Relativistic Electronic Structure Theory, Parts I and 2, ed. P. Schwerdtfeger (Elsevier, Amsterdam, 2002).

[13] BAND2016, SCM, Theoretical Chemistry, Vrije Universiteit, Amsterdam, The Netherlands, http://www. scm. com.

[14] P. Pyykkö, Phys. Chem. Chem. Phys. 14, 14734 (2012).

[15] P. Pyykkö, Chem. Rev. 112, 371 (2012).

[16] C.S. Nash and B.E. Bursten, J. Am. Chem. Soc. 121, 10830 (1999).

[17] V. Pershina and J. Anton, J. Chem. Phys. 138, 174301 (2013).

[18] J. Even et al., Science 345, 1491 (2014).

[19] R. Eichler et al., Nature, Letters 447, 72 (2007).

[20] R. Eichler et al., Radiochim. Acta 98, 133 (2010).

[21] A. Yakushev et al., Inorg. Chem. 53, 1624 (2014).

[22] N. Gaston et al., Angew. Chem. Int. Ed. 46, 1663 (2007).

[23] V. Pershina, J. Anton, T. Jacob, J. Chem. Phys. 131, 084713 (2009).

[24] V. Pershina, in progress.

[25] V. Pershina, Phys. Chem. Chem. Phys. 18, 17750 (2016). 